Case Report

\title{
May-Thurner Syndrome in Pregnancy: A Case Report
}

\author{
Olufemi Ajibade ${ }^{1}$, Radhika Malhotra ${ }^{2}$, Lamarr Tyler ${ }^{1}$, Siri Kjos ${ }^{1}$, David Howard ${ }^{2,}$ \\ ${ }^{1}$ Sunrise Health GME Health Consortium Residency Program Obstetrics and Gynecology, Las Vegas, NV, \\ USA \\ ${ }^{2}$ Department of Obstetrics, Gynecology, and Reproductive Health, Rutgers New Jersey Medical School, New- \\ ark, NJ, USA \\ *Correspondence: dlh246@njms.rutgers.edu
}

\begin{abstract}
May-Thurner Syndrome, left iliac vein compression by the right iliac artery, is a congenital permissive lesion that requires an additional insult prior to the onset of symptoms. We present a case of a 31-year-old gravida 1 para 0 at 28.6 weeks who presented to the emergency room with a 2month history of progressive lower left leg swelling since 21.2 weeks' gestation. The patient endorsed incidence of domestic violence at 17 weeks' gestation. The patient had a twin sister who was also pregnant and had not experienced these symptoms. Physical exam revealed 3+ pitting edema in the left lower extremity from ankle to groin. There was left calf tenderness with negative Homan's sign bilaterally. Overall finding on magnetic resonance angiography revealed severe compression of the upper left common iliac vein and lower inferior vena cava by gravid uterus. Conservative management and anticoagulation is instrumental in preventing veno-thromboembolic events in pregnancies complicated by MTS.
\end{abstract}

Keywords: May-Thurner Syndrome, pregnancy, veno-thromboembolic events in pregnancy, anticoagulation during pregnancy

How to cite this paper: Ajibade, O., Malhotra, R., Tyler, L., Kjos, S., \& Howard, D. (2022). May-Thurner Syndrome in Pregnancy: A Case Report. Global Journal of Medical Case Reports, 2(1), 5-8. Retrieved from https://www.scipublications.com/journal/index.php/gjmcr/article/view/216

Received: December 28, 2021

Accepted: February 3, 2022

Published: February 4, 2022

Copyright: (c) 2022 by the authors. Submitted for possible open access publication under the terms and conditions of the Creative Commons Attribution (CC BY) license (http://creativecommons.org/licenses /by/4.0/).

\section{Introduction}

First described by May and Thurner in 1957, May-Thurner Syndrome is described as left iliac vein compression by the right common iliac artery, which can lead to increased risk of deep vein thrombosis or lymphedema [1]. There have been limited cases shown to be identified during pregnancy, mostly in the postpartum period and particularly with presentation of DVT partly owed to the hypercoagulable status of pregnancy [4-6]. We present a case of a 31-year-old woman presenting at 28 weeks' gestation with progressive history of lower left leg edema since 21 weeks' gestation. The primary objective of this study is to describe a case of MTS in a population where a gravid uterus can often exacerbate the compression of the iliac vein, causing it to be the initial time a patient is diagnosed with the disease.

\section{Case}

A 31-year-old G1P0 at 28.6 weeks presented to the ED with a 2-month history of progressive lower left extremity (LLE) swelling since 21.2 weeks' gestation. The patient had active range of motion with normal strength in all extremities and denied loss of sensation or pain. The patient had no history of DVT, pulmonary embolism, thrombophilia, or past history of leg swelling. The patient's twin sister was pregnant at the same time but did not exhibit these symptoms. The patient denied recent injury but stated that there was an incidence of domestic violence at 17 weeks' gestation. Physical exam revealed 3+ pitting edema in the LLE from ankle to groin. The left thigh measured 26 inches and the left calf 
measured 19 inches. Right extremity exhibited no edema, with thigh 22 1/2 inches and calf $16 \frac{1}{2}$ inches. There was left calf tenderness with negative Homan's sign bilaterally.

The initial workup included an outpatient venous Doppler ultrasound of bilateral lower extremities, which revealed no identified DVT. One additional outpatient Doppler ultrasound was obtained due to increasing swelling and was negative. The patient then presented to the emergency department (ED). A third Doppler ultrasound was obtained in the ED and was negative for DVT. An orthopedic consult ruled out compartment syndrome. One dose of Ancef was given prophylactically for possible cellulitis with no clinical improvement. A thorough thrombophilia workup was obtained and negative. Protein $\mathrm{C}$ and $\mathrm{S}$ were elevated (Table 1$)$.

Table 1. Workup values.

\begin{tabular}{|c|c|}
\hline Coagulation Test & Results \\
\hline PT/INR & 9.8 seconds/0.94 \\
\hline PTT & 26 seconds \\
\hline Factor V Leiden Mutation & Negative \\
\hline Anti-Thrombin III Antigen & $110 \mathrm{IU} / \mathrm{ml}$ \\
\hline Free Protein S Antigen & $40 \mathrm{IU} / \mathrm{ml}$ \\
\hline Free Protein S Activity & $58 \%$ (elevated) \\
\hline Protein C Antigen & $88 \mathrm{IU} / \mathrm{ml}$ \\
\hline Protein C Activity & 104 (elevated) \\
\hline
\end{tabular}

Magnetic resonance angiography of the pelvis without contrast revealed no good evidence for DVT of left pelvic veins, and there was also no evidence of pelvic mass compression. Figure 1 shows the right common iliac artery compressing the origin of the left common iliac vein, a finding that is compounded by the compression from the gravid uterus. On diagnosis, patient began Lovenox $40 \mathrm{mg}$ daily and was instructed to apply Ace bandage to the lower extremity until edema resolved. An abdominal binder was used to decrease uterine pressure and compression of pelvic veins. The patient was deemed stable for discharge with close outpatient follow up on hospital day 3.

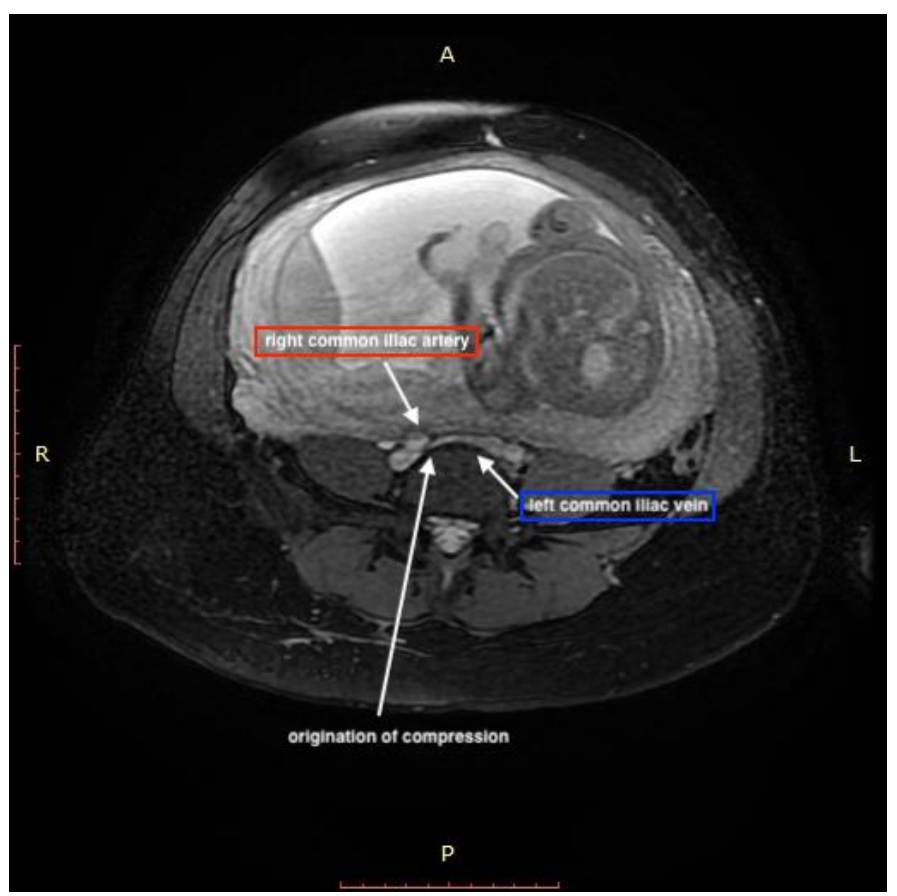

Figure 1. MR Angiography of the Pelvis 
Axial view - The right common iliac artery (as pointed out) is compressing the origin of the left common iliac vein. Gravid uterus is shown to exacerbate this compression as noted in the image.

One week after discharge, patient was seen in clinic with notable improvement in LLE swelling. Patient remained compliant with Lovenox during the pregnancy course with symptomatic improvement. Patient had spontaneous rupture of membranes at 39 weeks' gestation with last Lovenox dose administered the day before. The labor was augmented by Pitocin and the patient experienced uncomplicated spontaneous vaginal delivery to a live-born female weighing 3,110 grams, Apgars 7/8 with tight nuchal cord and an estimated blood loss of $300 \mathrm{~mL}$ during delivery. Lovenox was resumed on postpartum day (PPD) 1. Patient developed preeclampsia with severe features with blood pressure criteria on PPD 2, treated with 24 hours of magnesium sulfate and labetalol $200 \mathrm{mg}$ BID. Patient was discharged on PPD 3 on labetalol and instructed to continue Lovenox $40 \mathrm{mg}$ daily for 6 weeks. At the 8-week postpartum visit, the patient was normotensive with no notable edema on exam. Two years since delivery, the patient remains in our care and has not developed any venous thromboembolism (VTE) since diagnosis.

\section{Discussion}

We present a novel case of May-Thurner syndrome (MTS) in a pregnant female without evidence of a DVT or previously identified risk factors. We believe this case is particularly novel considering our patient had an identical twin sister who was pregnant at 35 weeks' gestation at the time of presentation who had not experienced these symptoms, indicating there are extrinsic factors contributing to the development of symptoms. Our patient experienced domestic violence at 17 weeks' gestation and her twin sister had no symptoms. Review of the literature shows limited cases discovered in pregnancy with no cases showing deep venous thrombosis as the presenting symptom [4-6]. The purpose of this case report to highlight the need for improving diagnostic criteria for MTS and prevention of venous thromboembolism in pregnancy.

MTS is defined as an extrinsic venous compression caused by the arterial system against bony structures in the ilio-caval territory, leading to the formation of venous spurs [1]. It is a congenital malformation that acts as a permissive lesion. Most patients with this malformation will remain asymptomatic and typically require an additional risk factor for disease development [2]. Incidence of disease is unknown. Typical clinical presentation includes acute extremity pain and edema, venous claudication, and chronic signs of venous insufficiency. Patients typically present with LLE swelling involving the entire limb. Risk factors include female sex, scoliosis, hypercoagulable state, and dehydration.

Further research needs to elicit causes of MTS and improve diagnosis methods. Currently, diagnosis depends on imaging, including duplex ultrasound, MR venography, or CT scans. A venogram is employed if clinical suspicion is high with negative workup. For mild symptoms, compression stockings, elevation, and lying on unaffected side are recommended. For moderate to severe symptoms, endovascular therapy [7,8]. Anticoagulation alone can decrease post-thrombotic syndrome to $<10 \%$ but is not recommended as primary therapy $[5,6]$. We propose conservative management with anticoagulation can be effective mode of treatment in pregnant patients to reduce VTE and encourage more research into treatment options of MTS in pregnancy.

Author Contributions: O.A., R.M., L.T., S.K., and D.H., have contributed to writing - original draft preparation, D.H. has contributed to writing - review and editing, supervision, project administration. All authors have read and agreed to the published version of the manuscript.

Funding: This research has received no additional funding.

Data Availability Statement: N/A. 
Acknowledgments: The authors wish to thank Jasmine Hankey for her editorial assistance.

Conflicts of Interest: The authors have no conflicts of interest.

\section{References}

[1] May R, Thurner J. The cause of the predominantly sinistral occurrence of thrombosis of the pelvic veins. Angiology 1957;8(5):419-27.

[2] Kibbe MR, Ujiki M, Goodwin AL, Eskandari M, Yao J, Matsumura J. Iliac vein compression in an asymptomatic patient population. J Vasc Surg 2004;39(5):937-43.

[3] Wu MK, Luo XY, Zhang FX. Incidence and Risk Factors of Deep Venous Thrombosis in Asymptomatic Iliac Vein Compression: A Prospective Cohort Study. Chin Med J (Engl) 2016;129(18):2149-52. doi: 10.4103/0366-6999.189918.

[4] Wax JR, Pinette MG, Rausch D, Cartin A. May-Thurner syndrome complicating pregnancy: a report of four cases. J Reprod Med 2014 May-Jun;59(5-6):333-6.

[5] Goto M, Miura S, Yamamoto T, Fukuda Y, Kuwano T, Kimura I, et al. Anticoagulant Therapy in a Pregnant Woman with MayThurner Syndrome. Intern Med 2016;55(1):59-62. doi: 10.2169/internalmedicine.55.5215. Epub 2016 Jan 1.

[6] Nakajima Y, Masaoka N, Tsuzuki Y, Morooka M, Sakai M. May-Thurner syndrome resulting in acute iliofemoral deep vein thrombosis during the second trimester of pregnancy. J Obstet Gynaecol Res 2012 Aug;38(8):1106-10. doi: 10.1111/j.14470756.2011.01840.x. Epub 2012 Apr 30.

[7] Goto M, Miura S, Saku K. May-Thurner Syndrome: Authors' Reply. Intern Med 2016;55(15):2129-2129. doi:10.2169/internalmedicine.55.7249.

[8] Dasari M, Avgerinos E, Raju S, Tahara R, Chaer RA. Outcomes of iliac vein stents after pregnancy. J Vasc Surg Venous Lymphat Disord 2017 May;5(3):353-357. doi: 10.1016/j.jvsv.2017.01.013. 